\title{
Open-Access-Transformation in Linguistics
}

Open Access for excellent academic publications in the field of German Linguistics: Thanks to the support of 39 academic libraries, 9 frontlist publications from 2021 can be published as gold open access, without any costs to the authors.

The following institutions have contributed to the funding and thus promote the open access transformation in German linguistics and ensure free availability for everyone:

Universitätsbibliothek Augsburg

Universitätsbibliothek Bayreuth

University of California, Berkeley Library

Staatsbibliothek zu Berlin - Preußischer Kulturbesitz

Universitätsbibliothek der Freien Universität Berlin

Universitätsbibliothek der Humboldt-Universität zu Berlin

Universitätsbibliothek der Technischen Universität Berlin

Universitätsbibliothek Bielefeld

Universitäts- und Landesbibliothek Bonn

Staats- und Universitätsbibliothek Bremen

Universitätsbibliothek der Technischen Universität Chemnitz

Universitäts- und Landesbibliothek Darmstadt

Sächsische Landesbibliothek - Staats- und Universitätsbibliothek Dresden

Universitätsbibliothek Duisburg-Essen

Universitäts- und Landesbibliothek Düsseldorf

Universitätsbibliothek Eichstätt-Ingolstadt

Universitätsbibliothek Johann Christian Senckenberg, Frankfurt a. M.

Bibliothek der Pädagogischen Hochschule Freiburg

Niedersächsische Staats- und Universitätsbibliothek Göttingen

Universitätsbibliothek Greifswald

Universitätsbibliothek der FernUniversität in Hagen

Universitäts- und Landesbibliothek Sachsen-Anhalt, Halle (Saale)

Staats- und Universitätsbibliothek Hamburg Carl von Ossietzky

Gottfried Wilhelm Leibniz Bibliothek - Niedersächsische Landesbibliothek, Hannover

Universitäts- und Landesbibliothek Tirol, Innsbruck

Universitätsbibliothek Kassel - Landesbibliothek und Murhardsche Bibliothek der Stadt Kassel

Universitätsbibliothek der Universität Koblenz-Landau

Zentral- und Hochschulbibliothek Luzern

Bibliothek des Leibniz-Instituts für Deutsche Sprache, Mannheim

Universitätsbibliothek Marburg

Universitätsbibliothek der Ludwig-Maximilians-Universität München

Universitäts- und Landesbibliothek Münster

Bibliotheks- und Informationssystem der Carl von Ossietzky Universität Oldenburg

Universitätsbibliothek Osnabrück

Universitätsbibliothek Vechta

Herzog August Bibliothek Wolfenbüttel

Universitätsbibliothek Wuppertal

ZHAW Zürcher Hochschule für Angewandte Wissenschaften, Hochschulbibliothek

Zentralbibliothek Zürich 

Dieses Buch ist meiner Familie gewidmet - dem Teil, den ich schon seit einer ganzen Weile kenne und dem, den ich erst kürzlich gefunden habe. 
